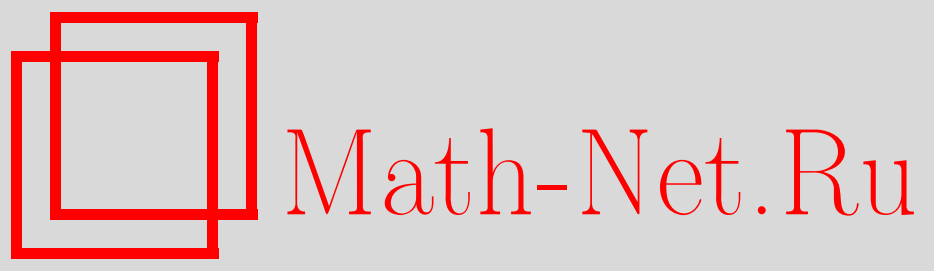

В. В. Вечернин, Корреляция между поперечными импульсами в модели со слиянием струн, ТМФ, 2015, том 184, номер 3, 437-448

DOI: https://doi.org/10.4213/tmf8929

Использование Общероссийского математического портала Math-Net.Ru подразумевает, что вы прочитали и согласны с пользовательским соглашением http://www.mathnet.ru/rus/agreement

Параметры загрузки:

IP: 3.93.64.190

26 апреля 2023 г., 07:57:42

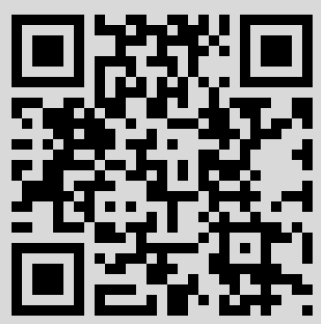




\title{
ФИЗИКА
}

Том 184, № 3

сентябрь, 2015

\section{КОРРЕЛЯЦИЯ МЕЖДУ ПОПЕРЕЧНЫМИ ИМПУЛЬСАМИ В МОДЕЛИ СО СЛИЯНИЕМ СТРУН}

\begin{abstract}
Получено явное аналитическое выражение для асимптотики коэффициента корреляции между средними поперечными импульсами частиц, образующихся в данном событии в интервалах, разнесенных по быстроте. Асимптотика найдена при большой плотности струн с учетом их слияния, путем введения решетки в поперечной плоскости и с использованием быстротной трансляционной инвариантности процесса распада струны при высоких энергиях. Установлено, что, в отличие от корреляции между импульсами отдельных частиц, величина корреляции между средними поперечными импульсами всех частиц, рождающихся в данном событии в двух разнесенных быстротных интервалах, не убывает с ростом общего числа образующихся струн, что делает этот тип корреляций перспективным для наблюдения в процессах столкновения ядер при высоких энергиях. Показано также, что в этом пределе асимптотика коэффициента корреляции между средними поперечными импульсами не зависит от величины дисперсии по числу частиц, образующихся при фрагментации струны, в отличие от изучавшихся ранее корреляций между множественностями или между средним поперечным импульсом и множественностью.
\end{abstract}

Ключевые слова: взаимодействия адронов, высокие энергии, множественное рождение, кварк-глюонные струны, корреляции поперечного импульса.

DOI: $10.4213 / \operatorname{tmf} 8929$

\section{1. ВВЕДЕНИЕ}

Анализ процессов рассеяния адронов при высоких энергиях показывает, что особенности сильного взаимодействия приводят к различным динамикам взаимодействия вдоль оси столкновения и в поперечной к ней плоскости. Как следствие, характеры спектров образующихся вторичных частиц по продольному $p_{z}$ и поперечному $\mathbf{p}_{\mathrm{t}}$ импульсам оказываются совершенно разными. При этом при описании их распределений по продольному импульсу оказывается удобнее перейти от продольной компоненты $p_{z}$ к быстроте

$$
y=\frac{1}{2} \ln \frac{p_{+}}{p_{-}}=\frac{1}{2} \ln \frac{p_{0}+p_{z}}{p_{0}-p_{z}} .
$$

* Санкт-Петербургский государственный университет, Санкт-Петербург, Россия. E-mail: v.vechernin@spbu.ru 
Еще до появления квантовой хромодинамики (КХД), в рамках подхода ГрибоваРедже [1] было осознано, что характерной чертой сильного взаимодействия является локальность взаимодействия партонов, образующих адроны, в пространстве быстрот, с одновременным ограничением величины их поперечного импульса. Было показано, что при описании процессов рассеяния адронов при высоких энергиях доминирует вклад от обмена помероном - специальным объектом, возникающим в рамках этого подхода. Позднее, подобная структура - известный померон Балицкого-Фадина-Кураева-Липатова [2], [3] - была получена и в рамках КХД как связанное состояние реджезованных глюонов.

Принцип локальности взаимодействия партонов в пространстве быстрот при высоких энергиях ведет к трансляционной инвариантности спектров рождающихся вторичных частиц, по крайней мере, в центральной области быстрот, вдали от крайних значений, отвечающих быстротам сталкивающихся адронов. В результате в этой области одночастичный спектр не зависит от быстроты $y$, а зависит только от поперечного импульса $\mathbf{p}_{\mathrm{t}}$ частицы. Двухчастичные распределения зависят лишь от разности быстрот $\Delta y=y_{1}-y_{2}$ и от поперечных импульсов $\mathbf{p}_{\mathrm{t}}^{1}$ и $\mathbf{p}_{\mathrm{t}}^{2}$.

Множественное рождение частиц при высоких энергиях в этом подходе описывается вкладом фейнмановских диаграмм с "разрезанием" померона. В работах [4], [5] была предложена пространственно-временная интерпретация этого процесса, соответствующая картине сильного взаимодействия в рамках КХД, которая позднее получила название иветного перецепления. В этой картине каждому "разрезанию" померона сопоставляется процесс формирования двух кварк-глюонных струн между партонами сталкивающихся адронов. Фрагментация этих струн позволяет описать процессы множественного рождения частиц при высоких энергиях. Тогда же была осознана необходимость учета вклада мультипомеронных процессов - процессов с обменом несколькими померонами. Вклад диаграммы с $N$ "разрезанными" померонами интерпретируется в этой картине как процесс взаимодействия адронов с образованием $2 N$ струн.

Описание процесса фрагментации образующихся струн в частицы за счет их разрыва при образовании кварк-антикварковых пар цветным полем струны строится как обобщение на случай КХД классического швингеровского механизма рождения $e^{+} e^{-}$-пар в сильном электрическом поле [6]. Имеется красивая, чисто геометрическая реализация алгоритма фрагментации струны для идеализированного случая струны Намбу [7]. Эти механизмы воспроизводят экспериментально наблюдаемое равномерное распределение вторичных частиц по быстроте в соответствии с ожидаемой трансляционной инвариантностью в центральной области быстрот. Подчеркнем, что использование этих алгоритмов во всех существующих монте-карловских генераторах событий, таких как PYTHIA, VENUS, HIJING, EPOS и др., является неизбежным, поскольку мягкие процессы, доминирующие во взаимодействиях адронов, пока не поддаются количественному описанию в рамках КХД.

Переход от рассеяния протонов к столкновениям тяжелых ядер при высоких энергиях приводит к образованию конфигураций с большой плотностью струн в поперечной плоскости. При этом возникает необходимость учета эффектов от наложения и взаимодействия первичных струн, с возможным их слиянием и образованием струнных кластеров, а также анализа влияния этих процессов на фрагментацию в наблюдаемые адроны. Как показано в работах [8]-[11], учет этих эффектов при- 
водит к значительному уменьшению общей множественности рождающихся частиц и увеличению их среднего поперечного импульса, что согласуется с имеющимися экспериментальными данными с коллайдеров RHIC и LHC [12], [13].

Более детальное изучение процессов взаимодействия и слияния струн можно проводить путем исследования влияния этих процессов на корреляции между наблюдаемыми величинами $F$ и $B$, измеренными для частиц с быстротами, лежащими в двух интервалах $\delta y_{F}$ и $\delta y_{B}$, разделенных некоторым быстротным зазором $y_{\text {gap }}$ [14]. В экспериментах на коллайдерах эти быстротные интервалы (окна), в которых регистрируют частицы, обычно соответствуют частицам, вылетающим в переднюю и заднюю полусферы, поэтому эти корреляции обычно называют корреляциями вперед-назад. Наличие быстротного зазора между окнами необходимо, так как оно позволяет разделить вклады так называемых ближних и дальних корреляций. Ближние корреляции возникают только между частицами, рождающимися при распаде одной и той же струны. Согласно трансляционной инвариантности они зависят лишь от разности быстрот частиц $\Delta y$. Локальность сильного взаимодействия в пространстве быстрот означает, что эти корреляции должны исчезать с увеличением $\Delta y$. Оценки показывают, что при выборе $y_{\text {gap }} \geqslant 1.5$ вкладом ближних корреляций между частицами с быстротами в интервалах $\delta y_{F}$ и $\delta y_{B}$ можно пренебречь. Остающиеся дальние корреляции возникают из-за синхронных флуктуаций от события к событию числа и вида струн, фрагментирующих в частицы с быстротами в интервалах $\delta y_{F}$ и $\delta y_{B}$.

В качестве величин $F$ и $B$, между которыми изучаются корреляции, обычно выбирают (см. [15], раздел 6.5.15, с. 1749) число заряженных частиц $n_{F}$ и $n_{B}$ с быстротами в интервалах $\delta y_{F}$ и $\delta y_{B}$ или их средние поперечные импульсы в данном событии

$$
p_{\mathrm{t}}^{F} \equiv \frac{1}{n_{F}} \sum_{i=1}^{M} p_{\mathrm{t}}^{i F}, \quad p_{\mathrm{t}}^{B} \equiv \frac{1}{n_{B}} \sum_{i=1}^{M} p_{\mathrm{t}}^{i B},
$$

где $p_{\mathrm{t}}^{i}=\left|\mathbf{p}_{\mathrm{t}}^{i}\right|$. В этом случае возможно изучение трех типов корреляций: $n-n, p_{\mathrm{t}}-n$ и $p_{\mathrm{t}}-p_{\mathrm{t}}$. Отметим, что для изучения возможных эффектов от слияния струн наиболее перспективным является исследование корреляций между средними событийными импульсами $p_{\mathrm{t}}^{F}$ и $p_{\mathrm{t}}^{B}$. Это связано с тем, что средний поперечный импульс является интенсивной переменной, в отличие от множественности, которая является экстенсивной величиной. Поэтому простые "объемные" флуктуации числа струн от события к событию (без их слияния), возникающие, например, из-за флуктуаций прицельного параметра, приводят к коррелированному изменению множественностей $n_{F}$ и $n_{B}$, но не приводят к корреляциям между $p_{\mathrm{t}}^{F}$ и $p_{\mathrm{t}}^{B}$. Для возникновения последних необходимо синхронное изменение качества источников в интервалах $\delta y_{F}$ и $\delta y_{B}$, например, вследствие различного слияния струн в каждом конкретном событии.

\section{2. ОБЩИЙ ФОРМАЛИЗМ}

Общий формализм описания дальних корреляций вперед-назад изложен в работах [16]-[18]. Вероятность одновременного наблюдения значений величин $F$ и $B$ в данном событии $P(F, B)$ может быть представлена в виде суммы по всевозможным 
конфигурациям $C$, характеризующим данное событие:

$$
P(F, B)=\sum_{C} P(C) P_{C}(F) P_{C}(B) .
$$

Вклад в (3) дают только дальние корреляции, так как для любой фиксированной конфигурации $C$ предполагается факторизация $P(F, B)$. Величина корреляции между наблюдаемыми $F$ и $B$ описывается корреляционной функцией $\langle B\rangle_{F}-$ регрессией, которая представляет собой условное среднее:

$$
\langle B\rangle_{F}=\frac{\sum_{C}\langle B\rangle_{C} P(C) P_{C}(F)}{\sum_{C} P(C) P_{C}(F)}=\frac{1}{P(F)} \sum_{C}\langle B\rangle_{C} P(C) P_{C}(F),
$$

и коэффициентом корреляции [15]

$$
\left.b_{B F} \equiv \frac{d\langle B\rangle_{F} /\langle B\rangle}{d F /\langle F\rangle}\right|_{F=\langle F\rangle}=\left.\frac{\langle F\rangle}{\langle B\rangle} \frac{d\langle B\rangle_{F}}{d F}\right|_{F=\langle F\rangle} .
$$

В формуле (4) знаменатель $P(F)=\sum_{C} P(C) P_{C}(F)$ имеет тот же вид, что и числитель, но без сомножителя $\langle B\rangle_{C}$. Поэтому ниже мы будем выписывать только преобразования числителя, подразумевая, что те же операции производятся и со знаменателем формулы (4).

В работах [12], [19], [20] были предложены различные варианты описания процесса слияния струн при формировании конфигурации $C$, возникающей в данном событии. Мы будем использовать самый простой вариант, предложенный в работах [17], [21] и состоящий в введении решетки в плоскости прицельного параметра с площадью ячейки порядка поперечного сечения струны. В этом случае возникающая в событии струнная конфигурация $C_{\eta}$ характеризуется набором целых чисел

$$
C_{\eta}=\left\{\eta_{1}, \ldots, \eta_{M}\right\}
$$

где $\eta_{i}$ - число центров струн, попавшее в $i$-ю ячейку, а $M$ - общее число ячеек. В определение конфигурации $C$, характеризующей данное событие, мы включим также $n_{i}^{F}$ и $n_{i}^{B}$ - числа частиц, образовавшихся от распада струн $i$-й ячейки в переднем и заднем быстротных интервалах:

$$
C=\left\{C_{\eta}, C_{n}^{F}, C_{n}^{B}\right\}, \quad C_{n}^{F}=\left\{n_{1}^{F}, \ldots, n_{M}^{F}\right\}, \quad C_{n}^{B}=\left\{n_{1}^{B}, \ldots, n_{M}^{B}\right\} .
$$

С учетом этого для вероятностей, входящих в выражение (4), имеем

$$
P(C)=P\left(C_{\eta}\right) P_{C_{\eta}}\left(C_{n}^{F}\right) P_{C_{\eta}}\left(C_{n}^{B}\right), \quad P_{C}(F)=P_{C_{\eta} C_{n}^{F}}(F) .
$$

Выражение для корреляционной функции теперь можно переписать так:

$$
\langle B\rangle_{F}=\frac{1}{P(F)} \sum_{C_{\eta}} P\left(C_{\eta}\right)\left[\sum_{C_{n}^{B}} P_{C_{\eta}}\left(C_{n}^{B}\right)\langle B\rangle_{C_{\eta} C_{n}^{B}}\right]\left[\sum_{C_{n}^{F}} P_{C_{\eta}}\left(C_{n}^{F}\right) P_{C_{\eta} C_{n}^{F}}(F)\right] .
$$

В качестве наблюдаемых величин $F$ и $B$, между которыми изучаются корреляции, следуя обсуждению в разделе 1 , мы выбираем средние поперечные импульсы $p_{F}$ и $p_{B}$ 
частиц, зарегистрированных в данном событии в каждом из быстротных окон (ниже мы опускаем индекс t):

$$
p_{F} \equiv \frac{1}{n_{F}} \sum_{i=1}^{M} \sum_{j=1}^{n_{i}^{F}} p_{i}^{j F}, \quad n_{F}=\sum_{i=1}^{M} n_{i}^{F},
$$

и аналогично для $p_{B}$ и $n_{B}$.

В соответствии с физической картиной слияния струн [20], [22] и интенсивным характером переменной, которой является поперечный импульс (см. раздел 1), средний поперечный импульс $\bar{p}_{i}$ частиц, образующихся при распаде $i$-й ячейки поперечного пространства, не зависит от числа образующихся частиц $n_{i}^{F}$ или $n_{i}^{B}$, а зависит только от степени перекрытия струн $\eta_{i}$ в этой $i$-й ячейке поперечного пространства. Поэтому он один и тот же для переднего и заднего наблюдательных окон. В этом случае, усредняя (10), мы имеем

$$
\left\langle p_{F}\right\rangle_{C_{\eta} C_{n}^{F}}=\frac{1}{n_{F}} \sum_{i=1}^{M} n_{i}^{F} \bar{p}_{i}\left(\eta_{i}\right)
$$

и аналогично для среднего $\left\langle p_{B}\right\rangle_{C_{\eta} C_{n}^{B}}$, входящего в правую часть (9).

\section{3. ГАУССОВО ПРИБЛИЖЕНИЕ}

Целью настоящей работы является нахождение асимптотики корреляционной функции $\left\langle p_{B}\right\rangle_{p_{F}}$ дальних $p_{\mathrm{t}}-p_{\mathrm{t}}$-корреляций при большой плотности струн. В этом случае для оценки вероятности $P\left(C_{\eta}\right)$ можно использовать гауссово приближение:

$$
P\left(C_{\eta}\right)=\prod_{i=1}^{M} \frac{1}{\sqrt{2 \pi D_{\eta_{i}}}} e^{-\left(\eta_{i}-\bar{\eta}_{i}\right)^{2} / 2 D_{\eta_{i}}}
$$

предполагающее независимые флуктуации числа струн $\eta_{i}$ в каждой ячейке вокруг заданного среднего значения $\bar{\eta}_{i}$ с некоторой дисперсией

$$
D_{\eta_{i}} \equiv \bar{\eta}_{i}^{2}-\bar{\eta}_{i}^{2} \sim \bar{\eta}_{i} \gg 1
$$

При этом сумма по всем струнным конфигурациям $C_{\eta}$, входящая в $(9)$ и представляющая собой многократную сумму по всем $\eta_{i}$, может быть заменена $M$-кратным интегрированием:

$$
\sum_{C_{\eta}}(\cdot)=\prod_{i=1}^{M} \sum_{\eta_{i}=0}^{\infty}(\cdot) \Rightarrow \prod_{i=1}^{M} \int_{0}^{\infty}(\cdot) d \eta_{i}
$$

Аналогичные приближения мы будем использовать и при суммировании по конфигурациям $C_{n}^{F}$ и $C_{n}^{B}(7)$ в формуле (9):

$$
P_{C_{\eta}}\left(C_{n}^{F}\right)=\prod_{i=1}^{M} \frac{1}{\sqrt{2 \pi D_{n_{i}^{F}}\left(\eta_{i}\right)}} e^{-\left[n_{i}^{F}-\bar{n}_{i}^{F}\left(\eta_{i}\right)\right]^{2} / 2 D_{n_{i}^{F}}\left(\eta_{i}\right)} .
$$


Важно, что в этом случае дисперсия $D_{n_{i}^{F}}$ и среднее число частиц $\bar{n}_{i}^{F}$ с быстротами в переднем окне $\delta y_{F}$, образующихся от распада струн в данной ячейке, зависят от числа струн $\eta_{i}$ в этой ячейке:

$$
D_{n_{i}^{F}}\left(\eta_{i}\right) \sim \bar{n}_{i}^{F}\left(\eta_{i}\right) \gg 1
$$

В силу симметрии для $P_{C_{\eta}}\left(C_{n}^{B}\right)$ выполняются аналогичные соотношения.

Наконец, в силу центральной предельной теоремы теории вероятностей, учитывая область переменных, задаваемую соотношениями (13) и (16), в которой мы ищем асимптотику корреляционной функции, для $P_{C_{\eta} C_{n}^{F}}\left(p_{F}\right)$ в формуле $(9)$ мы имеем

$$
P_{C_{\eta} C_{n}^{F}}\left(p_{F}\right)=\frac{1}{\sqrt{2 \pi D_{p_{F}}\left(C_{\eta}, C_{n}^{F}\right)}} e^{-\left[p_{F}-\left\langle p_{F}\right\rangle_{C_{\eta} C_{n}^{F}}\right]^{2} / 2 D_{p_{F}}\left(C_{\eta}, C_{n}^{F}\right)},
$$

где согласно (11) среднее этого распределения равно сумме средних $\bar{p}_{i}\left(\eta_{i}\right)$, деленной на $n_{F}$, а его дисперсия равна сумме дисперсий для отдельных частиц $D_{p_{i}}\left(\eta_{i}\right)$, деленной на $n_{F}^{2}$ :

$$
D_{p_{F}}\left(C_{\eta}, C_{n}^{F}\right)=\frac{1}{n_{F}^{2}} \sum_{i=1}^{M} n_{i}^{F} D_{p_{i}}\left(\eta_{i}\right)
$$

Напомним, что знаменатель $P(F)$ в формулах (4) и (9) отличается от числителя только отсутствием подынтегрального множителя $\langle B\rangle_{C}=\langle B\rangle_{C_{\eta} C_{n}^{B}}$, который в случае $p_{\mathrm{t}}-p_{\mathrm{t}}$-корреляций равен $\left\langle p_{B}\right\rangle_{C_{\eta} C_{n}^{B}}$ и задается соотношением, аналогичным (11). Поскольку мы ищем асимптотику корреляционной функции (9) в области, задаваемой соотношениями (13) и (16), то для оценки многомерных интегралов, возникающих в числителе и знаменателе формулы (4), мы будем использовать метод перевала. С этой целью, используя приведенные выше формулы, удобно представить корреляционную функцию (9) в следующем виде:

$$
\begin{aligned}
\left\langle p_{B}\right\rangle_{p_{F}}= & \frac{1}{P(F)} \prod_{i=1}^{M} \int_{0}^{\infty} d \eta_{i} d n_{i}^{F} N\left(C_{\eta}, C_{n}^{F}\right) e^{-\Phi\left(p_{F}, C_{\eta}, C_{n}^{F}\right)} \times \\
& \times \prod_{i=1}^{M} \int_{0}^{\infty} d n_{i}^{B} e^{-\Psi\left(C_{\eta}, C_{n}^{B}\right)}\left\langle p_{B}\right\rangle_{C_{\eta} C_{n}^{B}},
\end{aligned}
$$

где

$$
\Phi\left(p_{F}, C_{\eta}, C_{n}^{F}\right)=\sum_{i=1}^{M} \frac{\left(\eta_{i}-\bar{\eta}_{i}\right)^{2}}{2 D_{\eta_{i}}}+\sum_{i=1}^{M} \frac{\left[n_{i}^{F}-\bar{n}_{i}^{F}\left(\eta_{i}\right)\right]^{2}}{2 D_{n_{i}^{F}}\left(\eta_{i}\right)}+\frac{\left[p_{F}-\left\langle p_{F}\right\rangle_{C_{\eta} C_{n}^{F}}\right]^{2}}{2 D_{p_{F}}\left(C_{\eta}, C_{n}^{F}\right)}
$$

и

$$
\Psi\left(C_{\eta}, C_{n}^{B}\right)=\sum_{i=1}^{M} \frac{\left[n_{i}^{B}-\bar{n}_{i}^{B}\left(\eta_{i}\right)\right]^{2}}{2 D_{n_{i}^{B}}\left(\eta_{i}\right)} .
$$

Мы не выписываем явно предэкспоненциальный множитель $N\left(C_{\eta}, C_{n}^{F}\right)$, так как он входит как в числитель формулы (19), так и в ее знаменатель $P(F)$, и при вычислении их асимптотик методом перевала он сокращается. Так как знаменатель $P(F)$ 
в формуле (19) имеет такой же вид, как числитель, но без множителя $\left\langle p_{B}\right\rangle_{C_{\eta} C_{n}^{B}}$ под интегралом, то оценка интегралов по $n_{i}^{B}$ методом перевала в силу (21) приводит к простой замене $n_{i}^{B}$ на $\bar{n}_{i}^{B}\left(\eta_{i}\right)$ в подынтегральном выражении:

$$
\left\langle p_{B}\right\rangle_{p_{F}}=\frac{1}{P(F)} \prod_{i=1}^{M} \int_{0}^{\infty} d \eta_{i} d n_{i}^{F} N\left(C_{\eta}, C_{n}^{F}\right) e^{-\Phi\left(p_{F}, C_{\eta}, C_{n}^{F}\right)}\left\langle p_{B}\right\rangle_{C_{\eta} C_{n}^{B}},
$$

где, учитывая для $\left\langle p_{F}\right\rangle_{C_{\eta} C_{n}^{F}}$ формулу, аналогичную (11), мы имеем

$$
\left\langle p_{B}\right\rangle_{C_{\eta} C \frac{B}{n}}=\frac{1}{\bar{n}_{B}} \sum_{i=1}^{M} \bar{n}_{i}^{B}\left(\eta_{i}\right) \bar{p}_{i}\left(\eta_{i}\right), \quad \bar{n}_{B}=\sum_{i=1}^{M} \bar{n}_{i}^{B}\left(\eta_{i}\right) .
$$

В результате вычисление асимптотики корреляционной функции (22) сводится к нахождению зависимости положения точки перевала, $\eta_{i}=\eta_{i}^{*}\left(p_{F}\right)$ и $n_{i}^{F}=n_{i}^{* F}\left(p_{F}\right)$, от величины $p_{F}$ исходя из условий

$$
\frac{\partial \Phi\left(p_{F}, C_{\eta}, C_{n}^{F}\right)}{\partial \eta_{i}}=0, \quad \frac{\partial \Phi\left(p_{F}, C_{\eta}, C_{n}^{F}\right)}{\partial n_{i}^{F}}=0 .
$$

Далее, при вынесении предэкспоненциальных множителей из подынтегральных выражений в формуле $(22)$ в точке перевала $\eta_{i}^{*}$ и $n_{i}^{* F}$ множители $N\left(C_{\eta^{*}}, C_{n^{*}}^{F}\right)$ в числителе формулы $(22)$ и в ее знаменателе $P(F)$ сокращаются. Сокращаются также и сами остающиеся после вынесения предэкспонент интегралы. В результате для асимптотики функции $p_{\mathrm{t}}-p_{\mathrm{t}}$-корреляций мы находим

$$
\left\langle p_{B}\right\rangle_{p_{F}}=\left\langle p_{B}\right\rangle_{C_{\eta^{*}} C_{n}^{B}},
$$

где согласно (23)

$$
\left\langle p_{B}\right\rangle_{C_{\eta^{*}} C_{\frac{B}{n}}}=\frac{1}{\bar{n}_{B}} \sum_{i=1}^{M} \bar{n}_{i}^{B}\left(\eta_{i}^{*}\right) \bar{p}_{i}\left(\eta_{i}^{*}\right), \quad \bar{n}_{B}=\sum_{i=1}^{M} \bar{n}_{i}^{B}\left(\eta_{i}^{*}\right),
$$

а зависимость $\eta_{i}^{*}\left(p_{F}\right)$ задается положением точки перевала (24).

Теперь мы можем вычислить коэффициент $p_{\mathrm{t}}-p_{\mathrm{t}}$-корреляций согласно определению (5):

$$
\left.b_{p_{t} p_{\mathrm{t}}} \equiv \frac{d\left\langle p_{B}\right\rangle_{p_{F}}}{d p_{F}}\right|_{p_{F}=\left\langle p_{F}\right\rangle}=\left.\sum_{i=1}^{M} \frac{\partial}{\partial \eta_{i}^{*}}\left[\left\langle p_{B}\right\rangle_{C_{\eta^{*}} C_{\frac{B}{n}}}\right] \cdot \frac{d \eta_{i}^{*}}{d p_{F}}\right|_{p_{F}=\left\langle p_{F}\right\rangle} .
$$

Отметим, что для этого, как ясно из (27), нам нужно знать зависимость положения точки перевала $\eta_{i}^{*}\left(p_{F}\right)$ от $p_{F}$ лишь в малой окрестности вблизи $\left\langle p_{F}\right\rangle$.

Оценка методом перевала более простых интегралов по $\eta_{i}$, возникающих при вычислении самих средних величин $\left\langle p_{F}\right\rangle$ и $\left\langle p_{B}\right\rangle$, приводит к простой замене всех $\eta_{i}$ на $\bar{\eta}_{i}$ в формуле (23), аналогично тому, как это имело место при усреднении по конфигурациям $C_{n}^{B}$ в формуле (19) (см. комментарии после формулы (21)):

$$
\left\langle p_{F}\right\rangle=\left\langle p_{F}\right\rangle_{C_{\bar{\eta}} C_{\bar{n}}}, \quad\left\langle p_{B}\right\rangle=\left\langle p_{B}\right\rangle_{C_{\bar{\eta}} C_{\bar{n}}^{B}} .
$$

Поскольку все $\bar{n}_{i}^{F}\left(\eta_{i}\right) \sim \bar{n}_{i}^{B}\left(\eta_{i}\right)$, то согласно (23) справедливо равенство $\left\langle p_{F}\right\rangle=\left\langle p_{B}\right\rangle$, как и должно быть из общих соображений, приведенных после формулы (10). 


\section{4. АСИМПТОТИКА ДЛЯ ОДНОРОДНОГО СЛУЧАЯ}

В этом разделе мы реализуем программу, описанную выше, для простого однородного случая - с одинаковой средней плотностью струн во всей поперечной плоскости $\left(\bar{\eta}_{i}=\bar{\eta}\right)$ и одинаковой дисперсией $\left(D_{\eta_{i}}=D_{\eta}\right)$ относительно этого среднего. Удобно ввести так называемую приведенную дисперсию $\omega_{\eta}$ :

$$
\omega_{\eta}=\frac{D_{\eta}}{\bar{\eta}} .
$$

Мы также будем использовать конкретные зависимости среднего числа частиц $\bar{n}_{i}^{F}\left(\eta_{i}\right)$ и $\bar{n}_{i}^{B}\left(\eta_{i}\right)$, а также среднего поперечного импульса $\bar{p}_{i}\left(\eta_{i}\right)$ от степени перекрытия струн $\eta_{i}$ в данной ячейке, которые ожидаются в картине, основанной на КХД [8], [9], [11], [22]:

$$
\bar{n}_{i}^{F}\left(\eta_{i}\right)=\mu_{F} \sqrt{\eta_{i}}, \quad \bar{p}_{i}\left(\eta_{i}\right)=p_{0} \sqrt[4]{\eta_{i}}
$$

и аналогично для $\bar{n}_{i}^{B}\left(\eta_{i}\right)$. При этом, в соответствии с (16), мы также будем предполагать, что

$$
D_{n_{i}^{F}}\left(\eta_{i}\right)=\omega_{\mu} \bar{n}_{i}^{F}\left(\eta_{i}\right)
$$

с некоторой приведенной дисперсией $\omega_{\mu}$.

Коэффициенты $\omega_{\eta}$ и $\omega_{\mu}$ позволяют анализировать зависимость результирующего коэффициента $p_{\mathrm{t}}-p_{\mathrm{t}}$-корреляций при отклонении распределений по числу струн $\eta_{i}$ в данной ячейке и по числу частиц $n_{i}^{F}$ в переднем окне от распределения Пуассона, для которого $\omega_{\eta}$ и $\omega_{\mu}$ равны единице. Это необходимо, поскольку в реальных протон-протонных и ядро-ядерных столкновениях эти распределения могут значительно отличаться от пуассоновских [23], [24].

Наконец, для дисперсии импульса отдельной частицы $D_{p_{i}}$, рождающейся при распаде струнной конфигурации в $i$-й ячейке поперечного пространства, мы используем, основываясь на соображениях, приведенных после формулы (10), следующее выражение:

$$
D_{p_{i}}\left(\eta_{i}\right)=\gamma \bar{p}_{i}^{2}\left(\eta_{i}\right) .
$$

Отметим, что для обычно используемых распределений по поперечному импульсу $\rho(p)$ с одним размерным параметром, таких как $p e^{-p / \tilde{p}}, p e^{-p^{2} / \tilde{p}^{2}}, p(1+p / \tilde{p})^{-m}$, соотношение (32) всегда выполняется в силу соображений размерности. Безразмерный коэффициент $\gamma$ зависит только от вида распределения и имеет значение порядка единицы. При этом согласно (30) размерный параметр $\tilde{p}$, входящий в эти распределения, будет пропорционален $\sqrt[4]{\eta_{i}}[22],[25]$.

Подставляя выражения (30) и (32) в формулу (18) для дисперсии среднего импульса $p_{F}$ частиц, рожденных в данном событии, мы находим

$$
D_{p_{F}}\left(C_{\eta}, C_{n}^{F}\right)=\frac{\gamma p_{0}^{2}}{n_{F}^{2}} \sum_{i=1}^{M} n_{i}^{F} \sqrt{\eta_{i}}
$$

В результате для функции $\Phi\left(p_{F}, C_{\eta}, C_{n}^{F}\right)(20)$, определяющей согласно (24) положение точки перевала $\eta_{i}^{*}$ и $n_{i}^{* F}$, получаем следующее выражение:

$$
\Phi\left(p_{F}, C_{\eta}, C_{n}^{F}\right)=\frac{\bar{\eta}}{2 \omega_{\eta}}\left(\Phi_{1}+a \Phi_{2}+b \Phi_{3}\right),
$$


где

$$
\begin{gathered}
\Phi_{1}=\sum_{i=1}^{M}\left(z_{i}^{4}-1\right)^{2}, \quad \Phi_{2}=\sum_{i=1}^{M} \frac{\left(z_{i}^{2}-\rho_{i}\right)^{2}}{z_{i}^{2}} \\
\Phi_{3}=\left[\sum_{i=1}^{M} \rho_{i}\left(z_{i}-f\right)\right]^{2} / \sum_{i=1}^{M} \rho_{i} z_{i}^{2} .
\end{gathered}
$$

Здесь мы перешли от $\eta_{i}, n_{i}^{F}$ и $p_{F}$ к относительным переменным

$$
z_{i}^{4}=\frac{\eta_{i}}{\bar{\eta}}, \quad \rho_{i}=\frac{n_{i}^{F}}{\bar{n}_{i}^{F}(\bar{\eta})}, \quad f=\frac{p_{F}}{\left\langle p_{F}\right\rangle} .
$$

В рассматриваемом случае с однородным распределением струн в поперечной плоскости (29) согласно (30) и с учетом (23) и (28) мы имеем

$$
\bar{n}_{i}^{F}(\bar{\eta})=\mu_{F} \sqrt{\bar{\eta}}, \quad\left\langle p_{F}\right\rangle=\left\langle p_{F}\right\rangle_{C_{\bar{\eta}} C_{\bar{n}}^{F}}=\bar{p}_{i}(\bar{\eta})=p_{0} \sqrt[4]{\bar{\eta}}
$$

Значения констант $a$ и $b$ в (34) следующие:

$$
a=\frac{\omega_{\eta} \mu_{F}}{\omega_{\mu} \sqrt{\bar{\eta}}}, \quad b=\frac{\omega_{\eta} \mu_{F}}{\gamma \sqrt{\bar{\eta}}} .
$$

В новых переменных положение точки перевала $z_{i}=z_{i}^{*}(f), \rho_{i}=\rho_{i}^{*}(f)$ как функции $f$ согласно (24) и (34) определяется из условий

$$
\frac{\partial \Phi_{1}}{\partial z_{i}}+a \frac{\partial \Phi_{2}}{\partial z_{i}}+b \frac{\partial \Phi_{3}}{\partial z_{i}}=0, \quad \frac{\partial \Phi_{1}}{\partial \rho_{i}}+a \frac{\partial \Phi_{2}}{\partial \rho_{i}}+b \frac{\partial \Phi_{3}}{\partial \rho_{i}}=0 .
$$

Мы будем искать решение уравнений (40), не зависящее от $i$, исходя из симметрии рассматриваемого случая с однородным распределением струн в поперечной плоскости: $z_{i}=z, \rho_{i}=\rho$. В этом случае условия (40) принимают вид

$$
\begin{gathered}
4 z^{10}+4 z^{6}+a\left(z^{4}-\rho^{2}\right)+b \rho(z-f) f=0, \\
2 a\left(\rho-z^{2}\right)+b(z-f)^{2}=0 .
\end{gathered}
$$

Важно отметить, что в эти условия не вошло общее число ячеек в плоскости поперечного пространства $M$, тогда как сама функция $\Phi\left(p_{F}, C_{\eta}, C_{n}^{F}\right)(34)$ в этом случае пропорциональна $M$ :

$$
\Phi\left(p_{F}, C_{\eta}, C_{n}^{F}\right)=M \bar{\Phi}(f, z, \rho),
$$

что оправдывает применение метода перевала для оценки интеграла в формуле (22) при большом числе ячеек $M \gg 1$.

Уравнения (41) в рассматриваемом случае решают задачу нахождения функции $p_{\mathrm{t}}-p_{\mathrm{t}}$-корреляций $\left\langle p_{B}\right\rangle_{p_{F}}$, так как определяют, правда в неявной форме, зависимость положения точки перевала $z=z^{*}(f), \rho=\rho^{*}(f)$ от $f$. Действительно, согласно (25), (26) и (30) мы имеем

$$
\left\langle p_{B}\right\rangle_{p_{F}}=\left\langle p_{B}\right\rangle \cdot z^{*}(f), \quad f=\frac{p_{F}}{\left\langle p_{F}\right\rangle}
$$

где мы также учли (28), (37) и (38). 
Асимптотику же самого коэффициента $p_{\mathrm{t}}-p_{\mathrm{t}}$-корреляций $b_{p_{\mathrm{t}} p_{\mathrm{t}}}(27)$ в рассматриваемом случае удается найти в явном виде. В этом случае, как отмечалось выше, нам достаточно знать зависимость положения точки перевала $\eta_{i}^{*}\left(p_{F}\right)$ от $p_{F}$ лишь в малой окрестности вблизи $\left\langle p_{F}\right\rangle$. В новых переменных согласно (37) и (38) это отвечает окрестности $f=1$. В рассматриваемом однородном случае формула (27) принимает следующий простой вид:

$$
b_{p_{\mathrm{t}} p_{\mathrm{t}}}=\left.\frac{d z^{*}(f)}{d f}\right|_{f=1}=\left.z^{* \prime}(f)\right|_{f=1} .
$$

Эту производную при $f=1$ удается найти из уравнений (41) в явном виде, так как в окрестности $f=1$ эти уравнения линеаризуются.

Действительно, прежде всего легко заметить, что при $f=1$ уравнения (41) имеют решение $z=1$ и $\rho=1$, т. е. $\left.z^{*}(f)\right|_{f=1}=1$ и $\left.\rho^{*}(f)\right|_{f=1}=1$. Далее, дифференцируя (41) по $f$ и подставляя $f=1, z=1$ и $\rho=1$, находим

$$
\begin{gathered}
\left.16 z^{* \prime}(f)\right|_{f=1}+2 a\left[\left.2 z^{* \prime}(f)\right|_{f=1}-\left.\rho^{* \prime}(f)\right|_{f=1}\right]+b\left[\left.z^{* \prime}(f)\right|_{f=1}-1\right]=0, \\
a\left[\left.\rho^{* \prime}(f)\right|_{f=1}-\left.2 z^{* \prime}(f)\right|_{f=1}\right]=0 .
\end{gathered}
$$

Второе уравнение приводит к тому, что зависимость асимптотики от параметра $a$ уходит и остается лишь зависимость от $b(39)$. В результате для асимптотики коэффициента $p_{\mathrm{t}}-p_{\mathrm{t}}$-корреляций $b_{p_{\mathrm{t}} p_{\mathrm{t}}}$ в рассматриваемом случае с однородным распределением струн в поперечной плоскости (29) согласно (44) мы находим

$$
b_{p_{\mathrm{t}} p_{\mathrm{t}}}=\frac{b}{b+16}=\frac{\omega_{\eta} \mu_{F}}{\omega_{\eta} \mu_{F}+16 \gamma \sqrt{\bar{\eta}}} .
$$

\section{5. ОБСУЖДЕНИЕ РЕЗУЛЬТАТОВ}

Найденное выражение (46) для асимптотики коэффициента корреляции между средними поперечными импульсами в разнесенных быстротных интервалах при большой плотности струн необходимо сравнить с полученными ранее [17], [26] асимптотиками коэффициентов $n$ - $n$ - и $p_{\mathrm{t}}$ - $n$-корреляций:

$$
\begin{aligned}
b_{n n} & =\frac{a}{a+4}=\frac{\omega_{\eta} \mu_{F}}{\omega_{\eta} \mu_{F}+4 \omega_{\mu} \sqrt{\bar{\eta}}}, \\
b_{p_{t} n} & =\frac{1}{2} \frac{a}{a+4}=\frac{1}{2} \frac{\omega_{\eta} \mu_{F}}{\omega_{\eta} \mu_{F}+4 \omega_{\mu} \sqrt{\bar{\eta}}},
\end{aligned}
$$

а также с асимптотикой для коэффициента дальних $n$ - $n$-корреляций $\bar{b}_{n n}$, найденной в других подходах [21], [24] без учета эффектов от слияния струн:

$$
\bar{b}_{n n}=\frac{\omega_{\eta} \mu_{F}}{\omega_{\eta} \mu_{F}+\omega_{\mu}} .
$$

Заметим, что выражения (47) для $b_{n n}$ и $b_{p_{\mathrm{t}} n}$, конечно, получаются и в рамках изложенного в настоящей работе подхода. Вычисления в этих случаях значительно проще. При нахождении корреляционных функций $\left\langle p_{B}\right\rangle_{n_{F}}$ и $\left\langle n_{B}\right\rangle_{n_{F}}$ в формуле (34) отсутствует слагаемое $\Phi_{3}$, а минимум оставшегося выражения $\Phi_{1}+a \Phi_{2}$ по $\eta_{i}$ и $n_{i}^{F}$ 
необходимо искать при условии $\sum_{i=1}^{M} n_{i}^{F}=n_{F}$, которое легко учесть, вводя множитель Лагранжа.

Отличительной чертой полученного выражения (46) для коэффициента $p_{\mathrm{t}}-p_{\mathrm{t}}$-корреляции от всех других является его независимость от $\omega_{\mu}$, т. е. от величины дисперсии по числу частиц, образующихся при фрагментации струны.

Из (46) видно также, что найденный коэффициент $p_{\mathrm{t}}-p_{\mathrm{t}}$-корреляции между средними поперечными импульсами всех частиц, рождающихся в данном событии в двух разнесенных быстротных интервалах, не зависит от общего числа ячеек $M$ и, следовательно, от общего числа струн $N=\sum_{i=1}^{M} \eta_{i}, \bar{N}=M \bar{\eta}$, а зависит только от их средней плотности $\bar{\eta}$. Последнее выгодно отличает его от коэффициента корреляции между импульсами отдельных частиц, величина которого убывает пропорционально общему числу образующихся струн [17], [22], достигающему нескольких тысяч для центральных ядро-ядерных столкновений при высоких энергиях. Это наблюдение, с учетом сделанных в разделе 1 замечаний об интенсивном характере величины $p_{\mathrm{t}}$, делает именно этот тип корреляций перспективным для экспериментального изучения на ядерных коллайдерах RHIC и LHC [15] с целью обнаружения возможных эффектов от слияния цветных струн.

Благодарности. Автор благодарен организаторам конференции "В поисках фундаментальных симметрий", посвященной 90-летию со дня рождения Ю. В. Новожилова. Работа была поддержана РФФИ (грант № 15-02-02097) и Санкт-Петербургским государственным университетом (грант № 11.38.197.2014).

\section{Список литературы}

[1] В.Н.Грибов, ЖЭЭТФ, 53:2 (1967), 654-672.

[2] Э.А. Кураев, Л.Н. Липатов, В.С. Фадин, ЖЭТФ, 71 (1976), 840-855; 72 (1977), 377-389.

[3] Я. Я. Балицкий, Л.Н.Липатов, ЯФ, 28:2 (1978), 1597-1611.

[4] A. B. Kaidalov, Phys. Lett. B, 116:6 (1982), 459-463.

[5] A. Capella, U. Sukhatme, C.-I. Tan, J. Tran Thanh Van, 81:1 (1979), 68-74.

[6] J. Schwinger, Phys. Rev., 82:5 (1951), 664-679.

[7] X. Artru, Phys. Rept., 97:2-3 (1983), 147-171.

[8] T.S. Biro, H. B. Nielsen, J. Knoll, Nucl. Phys. B, 245 (1984), 449-468.

[9] A. Bialas, W. Czyz, Nucl. Phys. B, 267:1 (1986), 242-252.

[10] M. A. Braun, C. Pajares, Phys. Lett. B, 287:1-3 (1992), 154-158.

[11] M. A. Braun, C. Pajares, Nucl. Phys. B, 390:2 (1993), 542-558.

[12] M. A. Braun, F. del Moral, C. Pajares, Phys. Rev. C, 65:2 (2002), 024907, 4 pp., arXiv: hep-ph/0105263.

[13] K. Aamodt, B. Abelev, A.Abrahantes Quintana et al. [ALICE Collab.], Phys. Rev. Lett., 105:25 (2010), 252301, 11 pp., arXiv: 1011.3916.

[14] N. S. Amelin, N. Armesto, M. A. Braun, E. G. Ferreiro, C. Pajares, Phys. Rev. Lett., 73:21 (1994), 2813-2816.

[15] B. Alessandro, F. Antinori, J. A. Belikov et al. [ALICE Collab.], J. Phys. G, 32:10 (2006), 1295-2040.

[16] M. A. Braun, C. Pajares, V. V. Vechernin, Phys. Lett. B, 493:1-2 (2000), 54-64, arXiv: hep-ph/0007241.

[17] M. A. Braun, R. S. Kolevatov, C. Pajares, V. V. Vechernin, Eur. Phys. J. C, 32:4 (2004), 535-546, arXiv: hep-ph/0307056. 
[18] В. В. Вечернин, Р. С. Колеватов, ЯФ, 70:10 (2007), 1846-1857.

[19] N. S. Amelin, M. A. Braun, C. Pajares, Phys. Lett. B, 306:3-4 (1993), 312-318.

[20] M. A. Braun, C. Pajares, Eur. Phys. J. C, 16:2 (2000), 349-359, arXiv: hep-ph/9907332.

[21] В. В. Вечернин, Р.С. Колеватов, Вестн. С.-Петерб. ун-та. Сер. 4. Физика. Химия, 2004, вып. 2, 12-23, arXiv: hep-ph/0304295.

[22] M. A. Braun, C. Pajares, Phys. Rev. Lett., 85:23 (2000), 4864-4867, arXiv: hep-ph/0007201.

[23] V. V. Vechernin, H. S. Nguyen, Phys. Rev. C, 84:5 (2011), 054909, 12 pp., arXiv: 1102.2582.

[24] V. Vechernin, Nucl. Phys. A, 939 (2015), 21-45, arXiv: 1210.7588.

[25] В. В. Вечернин, Р. С. Колеватов, ЯФ, 70:10, 1858-1867.

[26] В. В. Вечернин, Р. С. Колеватов, Вестн. С.-Петерб. ун-та. Сер. 4. Физика. Химия, 2004, вып. 4, 11-27, arXiv: hep-ph/0305136. 